\title{
Auditory spatial representations of the world are compressed in blind humans
}

\author{
Andrew J. Kolarik ${ }^{1,2,3} \cdot$ Shahina Pardhan ${ }^{2} \cdot$ Silvia Cirstea $^{2}$ Brian C. J. Moore ${ }^{1}$
}

Received: 8 June 2016 / Accepted: 5 November 2016 / Published online: 11 November 2016

(C) The Author(s) 2016. This article is published with open access at Springerlink.com

\begin{abstract}
Compared to sighted listeners, blind listeners often display enhanced auditory spatial abilities such as localization in azimuth. However, less is known about whether blind humans can accurately judge distance in extrapersonal space using auditory cues alone. Using virtualization techniques, we show that auditory spatial representations of the world beyond the peripersonal space of blind listeners are compressed compared to those for normally sighted controls. Blind participants overestimated the distance to nearby sources and underestimated the distance to remote sound sources, in both reverberant and anechoic environments, and for speech, music, and noise signals. Functions relating judged and actual virtual distance were well fitted by compressive power functions, indicating that the absence of visual information regarding the distance of sound sources may prevent accurate calibration of the distance information provided by auditory signals.
\end{abstract}

Keywords Blindness $\cdot$ Spatial hearing $\cdot$ Auditory distance $\cdot$ Multisensory plasticity $\cdot$ Sound localization

Andrew J. Kolarik

ak771@cam.ac.uk

http://hearing.psychol.cam.ac.uk

1 Department of Psychology, University of Cambridge, Downing Street, Cambridge CB2 3EB, UK

2 Vision and Eye Research Unit (VERU), Postgraduate Medical Institute, Anglia Ruskin University, YST 215, Young Street, Cambridge CB1 1PT, UK

3 Centre for the Study of the Senses, Institute of Philosophy, University of London, Senate House, Malet Street, London WC1E 7HU, UK

\section{Introduction}

The ability to produce, retain, and update an accurate internal representation of the external world in the absence of a primary sensory modality is an important topic in psychology and neuroscience, and a large number of studies have attempted to address this by investigating the effects of visual loss on the ability to perform spatial tasks using auditory cues (for reviews, see Collignon et al. 2009; Voss et al. 2010). Accurate spatial representations based on sound are particularly important for blind people, as they underlie successful navigation performance (for a review, see Schinazi et al. 2016). It is clear that blind listeners do have a sense of auditory space. However, how this space is calibrated and how the calibration is maintained are not clear. The sense of auditory space for sounds within grasping distance may be calibrated via haptic feedback. For more distant sounds, it is possible that audiomotor feedback (the use of systematic changes in auditory stimuli resulting from self-motion) can be used to provide accurate calibration of auditory space in the absence of vision (Jones 1975; Ashmead et al. 1989; Lewald 2013). For example, rotation of the head about its vertical axis leads to corresponding changes in interaural time delay (ITD) and interaural level difference (ILD) cues for azimuthal localization, and the correspondence between the two might serve to calibrate the ITD and ILD cues (O'Regan and Noë 2001; Lewald 2002a).

The perceptual deficiency hypothesis posits that without vision to aid in calibrating audition, auditory spatial abilities may be poorer for those with visual loss than for sighted individuals (Axelrod 1959; Jones 1975). An opposing viewpoint is that blind individuals would have enhanced spatial abilities using sound due to extensive experience in extracting information from sound and reliance on sound 
(Rice 1970) and because compensatory processes, such as cortical reorganization, may enhance auditory spatial performance in certain conditions (Voss and Zatorre 2012). Both viewpoints have received support in the literature (for a review, see Voss et al. 2010). Some studies, mainly focusing on the ability to localize in azimuth or to detect changes in the azimuth or distance of sounds, have shown that blind listeners have sound localization abilities similar to or better than those for normally sighted listeners (Lessard et al. 1998; Doucet et al. 2005). However, blind individuals performed more poorly than sighted controls in an auditory spatial bisection task in which subjects reported whether the second sound of three sounds was closer to the left (first) or right (third) sound (Gori et al. 2014; Vercillo et al. 2016). Vertical sound localization has been reported to be less accurate for blind than for sighted listeners in both quiet conditions (Lewald 2002b) and in the presence of background noise (Zwiers et al. 2001). Evidence from Voss et al. (2015) suggests that a trade-off may occur between the horizontal and vertical planes for blind participants localizing sounds monaurally, such that learning to utilize monaural spatial cues for horizontal localization may come at the cost of using the monaural cues to localize sounds in terms of elevation.

The conditions under which visual loss leads to enhancement or worsening in auditory spatial perception have not been fully established. In particular, little is known about how visual loss affects the perception of distance using auditory cues. The aim of the current study was to compare the fidelity of the spatial representation of distance in extrapersonal space (farther than $1 \mathrm{~m}$ from the listener) for blind and normally sighted listeners using virtual auditory cues alone. The two main distance cues for stationary sounds in extrapersonal space are sound level (Coleman 1963; Mershon and King 1975) and direct-to-reverberant energy ratio, or D/R (Mershon and King 1975; Zahorik 2002a). Level generally provides more accurate information about distance [see Kolarik et al. (2016a) and Zahorik et al. (2005) for reviews]. However, if the room reverberation time is sufficiently long, the two cues can be equally effective (Kolarik et al. 2013a). Blind listeners usually show supra-normal performance for relative auditory distance judgments (Ashmead et al. 1998; Voss et al. 2004; Kolarik et al. 2013b), but a deficit in relative auditory distance judgments by both early-blind children and adults was reported by one study (Cappagli et al. 2015).

Absolute judgments of distance to single, static sources necessitate the use of a topographic representation of the auditory world without any reference or comparison sound sources. For normally sighted listeners, the visual system provides more accurate distance information than the auditory system (Da Silva 1985; Loomis et al. 1998), and visual information would normally be used to fine-tune neural representations of distance and to calibrate auditory information about distance. Lack of visual information, therefore, may lead to lower accuracy of absolute distance judgments by blind listeners if audiomotor feedback is not sufficient to calibrate auditory distance, consistent with the perceptual deficiency hypothesis. This viewpoint has been supported by studies showing that absolute auditory distance perception was less accurate for early-onset blind than for sighted participants, both for $800-\mathrm{Hz}$ tones (Wanet and Veraart 1985) and for white noises (Macé et al. 2012). It is currently unclear whether room reverberation affects absolute distance judgments for the blind, as the room reverberation time was not reported for these studies. Also, judgments were limited to sound sources in peripersonal space (sounds within reaching and grasping distance, up to approximately $1 \mathrm{~m}$ away from the individual). Another study showed that blind participants were less accurate than sighted participants at judging the distance of speech sounds simulated to be in extrapersonal space (Kolarik et al. 2013c). However, this experiment was conducted using a virtual anechoic room only, and room reverberation was not investigated. There is currently a gap in knowledge regarding the effect of visual loss on absolute distance judgments in extrapersonal space for different acoustic environments and for stimuli other than speech.

The current study used virtualization methods to investigate whether absolute distance judgments for virtual sounds in extrapersonal space would be less accurate for early-blind participants than for sighted controls, consistent with the perceptual deficiency hypothesis, and whether this generalized across anechoic and reverberant virtual rooms, and for a range of stimuli (speech, music, and noise, which differ in their spectro-temporal characteristics). The virtualization methods used in the current study allowed control over stimulus parameters including room reverberation time. To our knowledge, this is the first time that absolute distance judgments for blind and sighted individuals in both virtual anechoic and reverberant rooms have been assessed.

\section{Materials and methods}

\section{Participants}

There were two groups of participants: early-onset blind (defined here as having lost their sight between birth and 5 years of age, $n=10,4$ males and 6 females, mean age 45 years, range 25-69 years; see Table 1 for details) and normally sighted ( $n=11,5$ males and 6 females, mean age 41 years, range 20-67 years). The blind participants were either totally blind or had some light perception only, and fell into categories 4-5 of the World Health Organization 
Table 1 Details of blind participants

\begin{tabular}{llll}
\hline & $\begin{array}{l}\text { Sex, age, age of onset of } \\
\text { vision loss (years) }\end{array}$ & Cause of vision loss & Visual status, WHO category \\
\hline B1 & M, 46, 5 & Stickler's syndrome, retinal detachment & No light perception, 5 \\
B2 & F, 52, 5 & Macular degeneration & Light perception, 4 \\
B3 & M, 62, birth & Retinopathy of prematurity & No light perception, 5 \\
B4 & M, 25, 3 & Retinoblastoma & No light perception, 5 \\
B5 & M, 38, birth & Familial exudative vitreoretinopathy & No light perception, 5 \\
B6 & F, 52, 1.5 & Glaucoma & No light perception, 5 \\
B7 & F, 26, & Norrie disease & Light perception, 4 \\
B8 & F, 42, 1 & Retinoblastoma & No light perception, 5 \\
B9 & F, 69, 3 & Glaucoma & Light perception, 4 \\
B10 & F, 42, birth & Retinopathy of prematurity & No light perception, 5 \\
\hline
\end{tabular}

classification (World Health Organization 1989). Sighted participants reported normal or corrected-to-normal vision. All participants had normal or near-normal hearing, defined as better-ear average (BEA) hearing threshold across the frequencies 500,1000, 2000, and $4000 \mathrm{~Hz}$ equal to or less than $25 \mathrm{~dB}$ HL, as measured using an AS608 Interacoustics audiometer following the procedure recommended by the British Society of Audiology (2011). Participants were paid for taking part. The experiments followed the tenets of the Declaration of Helsinki. Informed consent was obtained from all participants following an explanation of the nature and possible consequences of the study. The experiments were approved by the Anglia Ruskin University Ethics Panel.

\section{Apparatus and stimuli}

Signals were generated by an ESI UGM96 sound card, using a custom-written MATLAB script (Mathworks, Inc.) with a response interface on a Lenovo T420 ThinkPad laptop. Following previous studies that tested the auditory abilities of blind participants (Teng et al. 2012; Rowan et al. 2013), blind participants were tested in a quiet laboratory room or in a quiet room in their homes if they preferred. Four blind and four sighted controls were tested at their homes. Stimuli were presented via closed-back Sennheiser HDA200 headphones, which have a transducer mounted within a hard shell casing, providing about $30 \mathrm{~dB}$ of passive attenuation of ambient noise, to ensure that extraneous background noises were inaudible. Digital filtering was used to correct the frequency response of the headphones so as to simulate free-field presentation. The stimuli were generated using methods described in previous studies (Kolarik et al. 2013a, b, c, d). A large virtual room measuring $30 \times 35 \times 10 \mathrm{~m}$ was simulated using an image source model, or ISM (Lehmann and Johansson 2008). The virtual room was either anechoic, so that level cues only were available, or reverberant, so that level and $D / R$ cues were available. The reverberation time $\left(T_{60}\right.$, the time taken for the sound level to fall by $60 \mathrm{~dB}$ ) was $700 \mathrm{~ms}$, comparable to that used in a previous study (Zahorik 2002b). The ISM synthesizes a room impulse response (RIR) between a virtual source and receiver separated by a specified distance. Convolution of the RIR with a sound sample provides a virtual sample of the sound heard within the simulated room, at the specified virtual distance. The simulated position of the participant was in the near-left corner $1 \mathrm{~m}$ from each wall at $1 \mathrm{~m}$ height, facing forward into the room at $30^{\circ}$ relative to the long wall (Fig. 1). Stimuli were presented at $1 \mathrm{~m}$ height, at $0^{\circ}$ azimuth relative to the front of the head and at $0^{\circ}$ elevation.

The stimuli consisted of speech, music, and broadband noise. One speech stimulus was a sentence spoken at a conversational level by a male, randomly selected from the Bench-Kowal-Bamford corpus (Bench et al. 1979). The sentence was sampled at $22.05 \mathrm{kHz}$ and had a duration of $1.5 \mathrm{~s}$, chosen to match stimuli previously used in a distance discrimination study with sighted participants (Akeroyd et al. 2007). Music stimuli consisted of a 7.3-s segment of a jazz trio (piano, bass and drums) sampled at $22.05 \mathrm{kHz}$, previously used to investigate the influence of upper cutoff frequency on preferences for music using hearing aids (Moore et al. 2011). Noise stimuli were broadband (0.6$12 \mathrm{kHz}$ ) white noise bursts with 90-ms duration and 10-ms rise/fall time, sampled at $44.1 \mathrm{kHz}$, chosen to match stimuli previously used to investigate auditory distance discrimination by sighted and blind participants (Voss et al. 2004; Kolarik et al. 2013b).

The distances of the simulated sound sources were defined as spatial coordinates located in extrapersonal space (here defined as farther than $1 \mathrm{~m}$ ) located directly in front of the simulated participant position at 1.22, $1.72,2.44,3.45,4.88,6.90,9.75$, and $13.79 \mathrm{~m}$, based on distances used in a previous distance estimation study by Zahorik (2002a). Stimuli were spatially rendered by convolving a nonindividualized head-related transfer function 


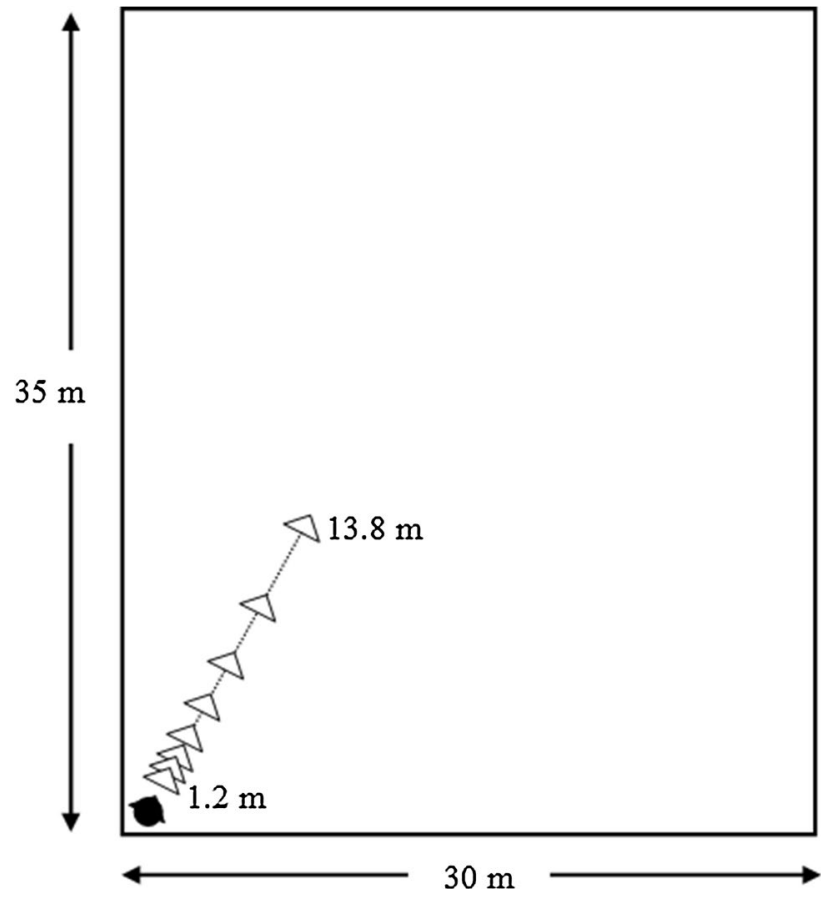

Fig. 1 Schematic of the virtual room and the configuration of the participant and sound sources. The participant's position is shown by the black symbol, and the simulated sound source positions are shown by open triangles

(HRTF) with the direct sound component. The HRTF was obtained from publicly available HRTF measurements made by Gardner and Martin (1995) using a Knowles Electronics Manikin for Acoustics Research under anechoic conditions for a distance of $1.4 \mathrm{~m}$ between the source and the manikin. Previous studies investigating spatial localization ability (Voss et al. 2011) and distance discrimination (Kolarik et al. 2013b) for blind participants also used this set of HRTF measurements. Otani et al. (2009) showed that HRTFs are approximately independent of source distance for distances greater than $1 \mathrm{~m}$. HRTFs measured at a fixed distance of $1 \mathrm{~m}$ have been used previously to study absolute distance judgments by sighted participants (Brungart and Scott 2001). Stimuli were processed offline before being saved in computer files for access during the experiment. The mean presentation level of the stimuli was $66 \mathrm{~dB}$ SPL (unweighted) for a virtual distance of $1 \mathrm{~m}$ from the participant's position. The level decreased with increasing virtual distance. For a discussion of the limitations of the methods used for simulation, see Kolarik et al. (2013b).

\section{Procedures}

Participants were asked to imagine themselves to be seated in a rectangular room of an unspecified size, listening to sounds emitted from a loudspeaker situated at various distances in front of them. The participants were informed that the stimuli would be simulated and that they should use only acoustic information regarding their perception of the environment. Sighted participants and blind participants with light perception were instructed to keep their eyes closed during the experiment, following Vercillo et al. (2015). ${ }^{1}$ They were monitored by the experimenter throughout the experiment to ensure that this instruction was followed. Single stimuli were presented in random order at the virtual distances listed above. Participants verbally reported the apparent distance of each sound source in meters and centimeters, or feet and inches if they preferred. Responses were recorded by the experimenter using the response interface. Participants were instructed to report zero meters or feet if they perceived the sound to originate within the head. No training was provided. No limitation was imposed on response time, and no feedback was given.

Within a single block of trials, stimulus type (speech, music or noise) and condition (anechoic or reverberant room) were kept constant. There were 10 repetitions per virtual distance, and thus 80 trials in each block. Measurements for the 3 stimulus types and 2 conditions were obtained within a single session ( 6 blocks $=480$ trials in total). The order of presentation of the 6 blocks was randomized. The experiment lasted approximately $1 \mathrm{~h}$ and $40 \mathrm{~min}$.

\section{Results}

\section{Auditory distance judgments}

No responses of zero distance to the sound source were reported by any of the participants, indicating that all

\footnotetext{
${ }^{1}$ A control experiment was conducted to assess whether performance differed between conditions where participants wore a blindfold and where they were instructed to keep their eyes closed. Normally sighted participants with normal hearing as described for the main experiment $(n=10,7$ males and 3 females, mean age 33 years, range $28-42$ years) were tested using reverberant speech only, as it was assumed that any effects of visual information would similarly affect auditory distance judgments for other stimuli. Half of the participants were blindfolded prior to entering the testing room. They then left the room and completed a distractor task (a questionnaire regarding general hearing abilities) for $15 \mathrm{~min}$. They then returned and were tested under instruction to keep their eyes closed, as for the main experiment. The other half of the participants completed the experiment in the reverse order. Similar performance was observed for the eyes closed and blindfolded conditions, and errors increased as virtual distance increased. Errors were evaluated using a within-subjects ANOVA with distance and vision occlusion method as factors. A main effect of distance only was found $[F(7,63)=16.67, p<0.001]$. Other main effects and interactions were not significant (all $p>0.05$ ), suggesting that participants followed the instructions to keep their eyes closed, and thus visual information did not affect performance.
} 
sounds were externalized. The accuracy of participants' distance judgments is shown in Fig. 2, which shows apparent distance judgments plotted as a function of virtual distance on $\log -\log$ coordinates. Open and filled symbols show geometric mean results for sighted and blind participants, respectively. Geometric means were used following Zahorik (2002a). The upper and lower panels show results for simulated reverberant and anechoic rooms, respectively. For each group (sighted or blind), results are shown separately for speech, music, and noise stimuli.

Zahorik et al. (2005) showed that compressive power functions of the form $r^{\prime}=k r^{a}$ gave good fits to normally sighted participants' judgments of distance using auditory cues, where $r^{\prime}$ is the estimate of perceived distance, $r$ is the actual source distance, and $k$ and $a$ are adjustable parameters. Slopes of linear fits to the current data on logarithmic

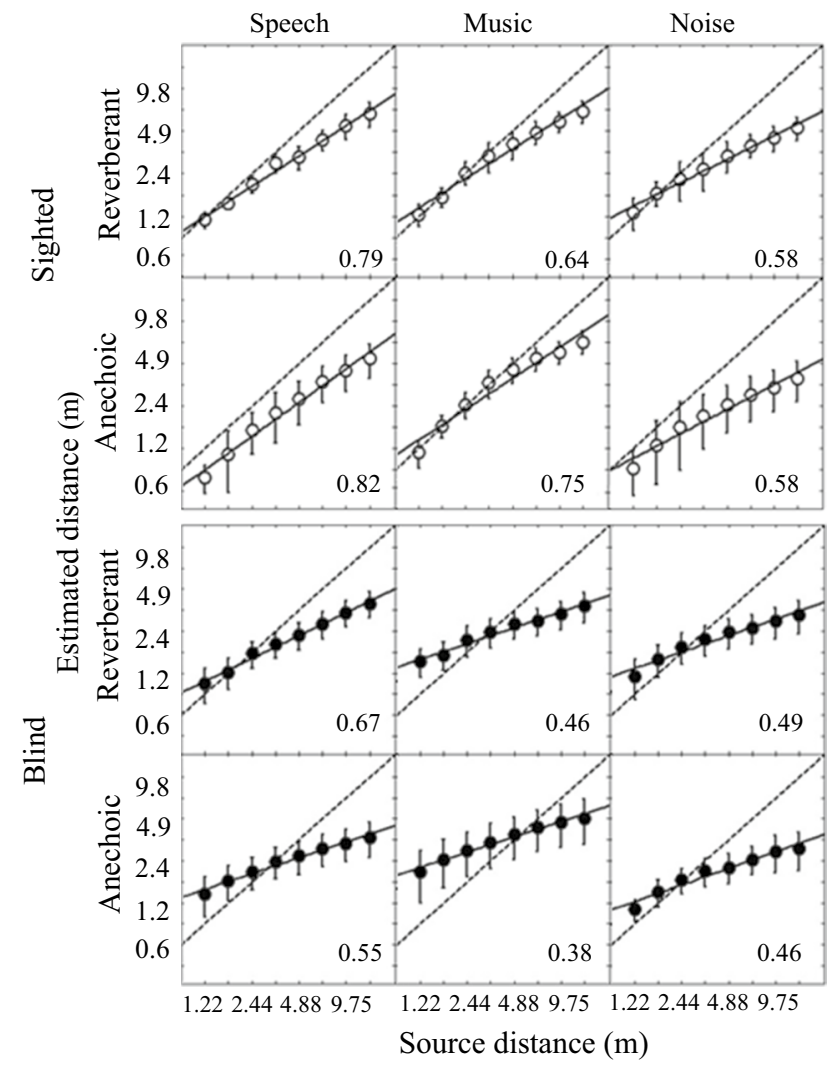

Fig. 2 Auditory distance judgments as a function of virtual source distance. Symbols show geometric mean data for sighted participants (upper six panels, open circles) and blind participants (lower six panels, filled circles). Results are shown in separate panels for speech, music, and noise stimuli. The upper and lower panels for each group show results for the simulated reverberant and anechoic rooms, respectively. Error bars indicate \pm 1 standard error across participants. Linear fits to the data on $\log -\log$ coordinates are shown by solid lines; the slope is reported in the bottom right corner of each panel (equivalent to the $a$ parameter of the compressive power function proposed by Zahorik et al. 2005). Dashed lines indicate where the points would lie if performance was perfect coordinates are equivalent to the $a$ parameter and are reported in the bottom right of each panel of Fig. 2.

For the normally sighted participants, the estimated distance was close to the actual distance for small distances but the distance was underestimated for greater distances, and the amount of underestimation increased as virtual distance increased. The slopes for speech stimuli were significantly steeper than those for noise stimuli [anechoic: $t(12)=4.54$, $p<0.001$, reverberant: $t(12)=5.24, p<0.001$ ], indicating more veridical distance judgments for speech sounds in both anechoic and reverberant simulated environments. The slope for music stimuli fell between those for the speech and noise stimuli, in both the anechoic and reverberant virtual rooms. For the blind participants, the distances to nearby sources were overestimated while the distances to farther sources were underestimated to a greater extent than for the sighted participants. Thus, the range over which distances were judged to change was markedly compressed relative to the actual range of distances. In the reverberant condition, the slope was significantly steeper for speech stimuli than for music $[t(12)=4.42, p<0.001]$ and noise $[t(12)=3.41, p<0.01]$ stimuli. Slopes for music and noise were similar in the reverberant condition. In the anechoic condition, the slope was steeper for speech stimuli than for music $[t(12)=2.63, p<0.05]$ but not noise $[t(12)=1.39$, $p<0.01]$. The slope was significantly less for the blind than for the sighted group in all but one of the conditions: speech [anechoic: $t(12)=3.63, p<0.01$; reverberant: $t(12)=2.82, p<0.05$ ], music [anechoic: $t(12)=5.24$, $p<0.001$, reverberant: $t(12)=3.89, p<0.001]$, and noise [anechoic: $t(12)=2.67, p<0.05$ ]. For noise in a reverberant virtual room, there was a trend in the same direction, but it was not significant $[t(12)=1.85, \mathrm{~ns}]$. A mixedmodel ANOVA of the slopes with reverberation time and stimulus as within-subjects factors and blindness as a between-subjects factor showed main effects of stimulus $[F(2,38)=6.60, p<0.01]$ and blindness $[F(1,19)=4.41$, $p<0.05]$. No other main effects or interactions were significant (all $p>0.05$ ). In summary, these findings indicate that blind participants' judgments of distance are compressed relative to those for sighted participants.

To examine the precision of distance judgments, the mean unsigned error (the difference between judged and virtual distance for each trial regardless of direction) was calculated. Figure 3 shows the mean errors for sighted and blind participants for each condition. The top, middle, and bottom panels show errors for speech, music, and noise, respectively. The left and right panels show errors for anechoic and reverberant rooms, respectively. For both groups, errors increased with increasing virtual distance, and judgments made in the reverberant room tended to be more accurate. Sighted participants tended to be more accurate than blind participants, especially for closer sound 
Fig. 3 Mean absolute errors of the distance judgments for sighted (open bars) and blind (gray bars) participants. The top, middle, and bottom panels show results for speech, music, and noise, respectively. The left and right panels show results for the simulated anechoic and reverberant rooms, respectively. Both axes are logarithmic. Error bars indicate \pm 1 standard error across participants

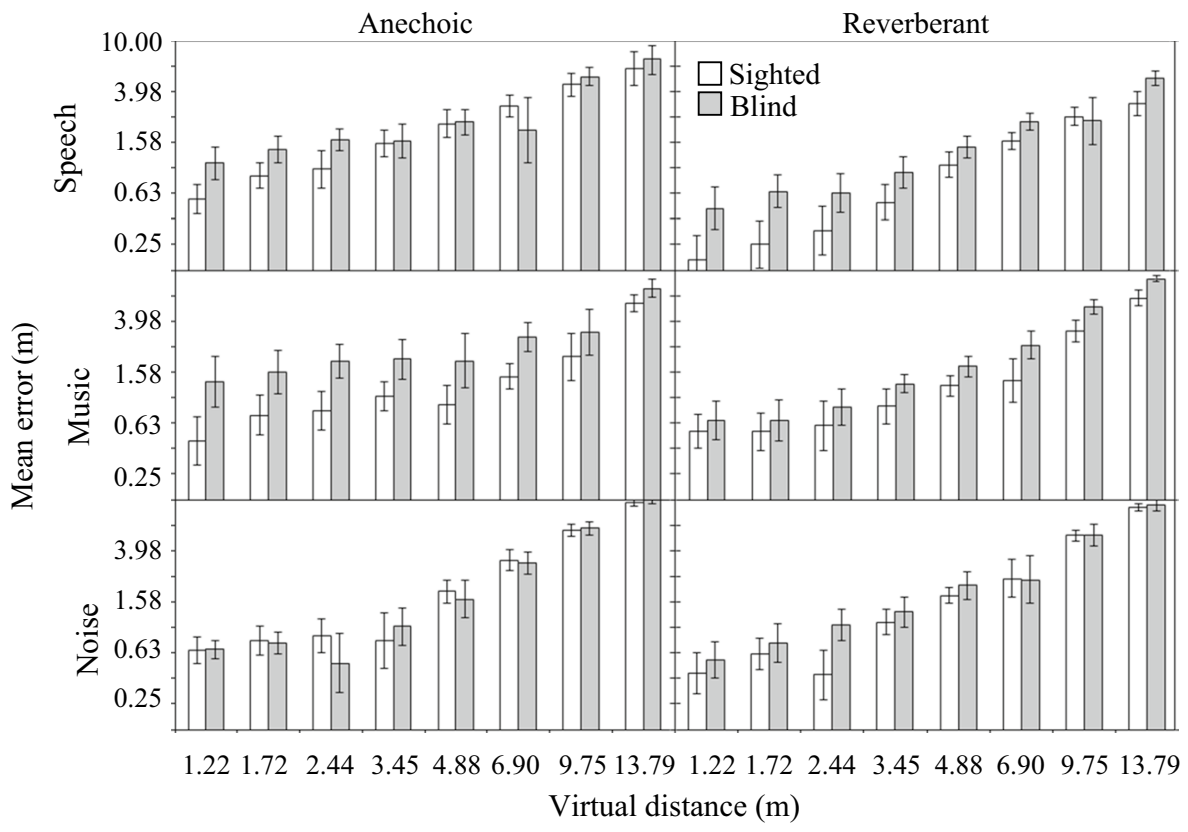

sources, and for speech in a reverberant virtual room and for music in an anechoic virtual room. A mixed-model ANOVA on the error scores with distance, reverberation time, and stimulus as within-subjects factors and blindness as a between-subjects factor showed main effects of distance $[F(7,133)=67.16, p<0.001]$, reverberation time $[F(1,19)=8.51, p<0.01]$, and blindness $[F(1,19)=5.56$, $p<0.05]$, and significant interactions between stimulus and reverberation time $[F(2,38)=3.72, p<0.05]$, and distance and reverberation time $[F(7,133)=3.03, p<0.01]$. No other main effects or interactions were significant (all $p>0.05)$. A posteriori tests with Bonferroni correction showed that error scores were significantly larger in the anechoic room than in the reverberant room for the speech stimulus only. In summary, the results show that errors were significantly higher for the blind than for the sighted participants.

The inherent variability of the judgments was assessed using the standard deviation (SD) of the ten judgments for each participant and each condition. A mixed-model ANOVA of the SDs, with distance, reverberation time, and stimulus as within-subjects factors and blindness as a between-subjects factor, showed main effects of stimulus $[F(2,38)=4.51, p<0.05]$ and distance $[F(7,133)=7.08$, $p<0.001]$, and interactions between blindness and distance $[F(7,133)=3.63, p<0.001]$, and stimulus and distance $[F(14,266)=1.91, p<0.05]$, but no main effect of blindness $[F(1,19)=4.23$, ns $]$. Although there was a trend for sighted participants to give higher judgment variability than blind participants at greater distances, a posteriori tests with Bonferroni correction showed no significant differences between the groups. Thus, the inherent variability of the distance judgments was not different for the blind and sighted participants.

\section{Subsidiary experiment: nonvisual, nonauditory distance judgments}

It is possible that the differences across groups described above were due to an underlying difference between blind and sighted participants in their ability to estimate distances in general. To assess this, we obtained distance judgments for a nonauditory and nonvisual task in which participants were instructed to walk forwards over distances of 2, 5, and $10 \mathrm{~m}$. Previous work has shown that for a visual distance task, both walked and verbal responses are controlled by the same internal variable of perceived distance and that walking responses are not regulated by another internal variable governed by the number of paces or walking time to reach objects (Philbeck and Loomis 1997). Assuming that this also applies to the nonvisual and nonauditory walking task in our subsidiary experiment, the results should give an indication of the general ability to judge perceived distance that can be compared to the perceived auditory distance judgments given by participants in the main experiments.

Groups consisted of blind ( $n=9$, including 6 participants who took part in the main experiment, 2 males and 7 females, mean age 44 years, range 22-69 years), and normally sighted participants $(n=9,5$ males and 4 females, mean age 39 years, range $22-67$ years). All participants had normal hearing, and blind participants had total visual loss or light perception only, as described for the main experiment. There were 3 repetitions for each of the predetermined distances of 2, 5, and $10 \mathrm{~m}$ (9 trials in total), and 


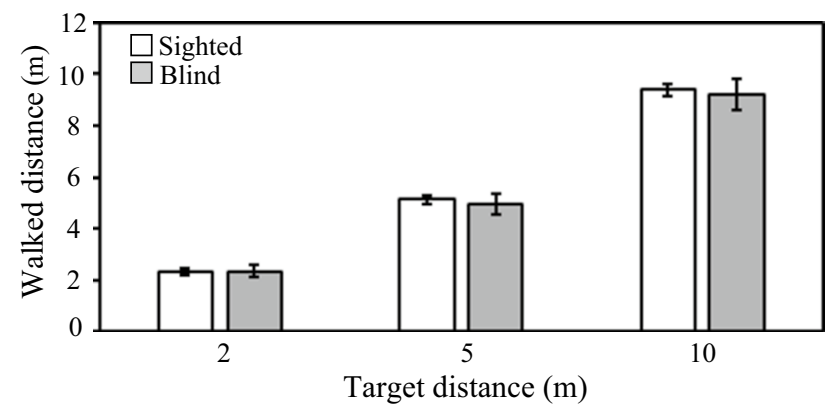

Fig. 4 Geometric mean walked distances for sighted participants (open bars) and blind participants (gray bars), plotted against the target distance. Error bars represent \pm 1 standard error

the order of the distances was randomized. For each trial, sighted participants and blind participants with light perception only were asked to close their eyes and walk forwards over the predetermined distance. No feedback was given.

Figure 4 shows geometric mean walked distances for sighted and blind participants. Walked distances were similar for sighted and blind participants for all three target distances. The mean unsigned error was assessed using a mixed-model ANOVA with distance as a within-subjects factor and blindness as a between-subjects factor. The main effect of distance was significant $[F(2,32)=4.35$, $p<0.05$ ], but there was no main effect of blindness or interaction (both $p>0.05$ ), suggesting that the differences across groups observed in the main experiment were not due to differences in general ability to judge distance between sighted and blind participants.

\section{Discussion}

Blind participants consistently overestimated the distance to nearby simulated sound sources and underestimated the distance to remote sound sources. They did the latter to a greater extent than sighted participants. These findings suggest that without visual information to aid in calibration, the internal representation of virtual auditory distance is compressed for both anechoic and reverberant environments, and across a range of stimuli, consistent with the perceptual deficiency hypothesis.

The judged distance data for sighted participants (Fig. 2) were consistent with effects described in the literature for both real and virtual sound sources, suggesting that the virtual techniques used here offered an acceptable simulation of auditory distance cues. Normally sighted participants consistently underestimated the distance to more distant sound sources, as reported for many studies of real and virtual apparent distance [see Kolarik et al. (2016a) and Zahorik et al. (2005) for reviews]. Judged distances tended to be more veridical for conversational-level speech stimuli (Gardner 1969) than for noise stimuli (Zahorik 2002a), consistent with the view that sighted participants can use their familiarity with the characteristics of speech to improve their judgments of apparent distance (Brungart and Scott 2001). However, it is possible that the longer duration of the speech stimuli contributed to the advantage observed here for those stimuli (the stimuli were chosen to match those used in previous studies and thus differed in duration). Although the simulation utilized nonindividualized HRTFs, it is unlikely that this contributed to the differences observed between blind and sighted participants, as pinna cues do not provide distance information for simulated stimuli placed directly ahead of the participant at distances greater than $1 \mathrm{~m}$ (Otani et al. 2009). It is therefore likely that the results of this study give a fair indication of distance judgments made by blind participants in real rooms. However, such judgments have yet to be obtained, and we confine discussion of the current findings to virtual environments only.

Given previous work showing that judgments of sound source azimuth by blind participants are as good as or better than those made by sighted participants, it appears that blind participants hear the direction of sound sources well, but make greater random and systematic errors than sighted participants in judging their distance. In the current study, lower precision was found for the closer sound sources for blind participants than for sighted controls. These results are in agreement with a study of relative auditory distance judgments by Cappagli et al. (2015), who found lower precision for blind than sighted controls for distances outside peripersonal space. The strong compression of auditory space for blind participants might be explained by a limited ability to calibrate distance using audiomotor feedback. Blind participants rarely, if ever, have direct information about the distance of sound sources in extrapersonal space in order to calibrate level and D/R cues. For sighted participants, visual range information about the whole scene increases the accuracy of auditory distance judgments even when the sound source itself is not visible (Zahorik 2001; Calcagno et al. 2012). As only limited range information is available to blind participants using audiomotor feedback, this may prevent the development of accurate internal representations of auditory distance. The current findings are consistent with previous work suggesting that visual information is needed to calibrate auditory space sufficiently accurately to allow good performance in more challenging auditory spatial tasks. For example, Gori et al. (2014) showed that although blind participants were well able to detect a change in azimuth, they displayed deficits when performing an auditory spatial bisection task requiring a representation of space that stayed in memory for a 
relatively long duration and necessitated the use of a wellcalibrated internal topographical spatial map.

It is possible that the point at which blind participants perceive auditory distance without bias correlates with information that they use to estimate distance in everyday life. For example, they may perceive that distance correctly because it correlates with the distance at which they tap with the cane in the floor. Although this was not investigated in the current study as some of our participants did not use a cane (instead relying on a guide $\operatorname{dog}$ ), further investigation would help to establish whether there is a correlation between point of correct perceived auditory distance for each participant and the distance at which they are used to tapping or generating sound with the cane on the floor, that could suggest that auditory perception can be calibrated by audiomotor feedback.

The compression of internal representations of distance following visual loss may have consequences for navigation. According to representation or model-based control approaches (Frenz and Lappe 2005; Turano et al. 2005), sensory information allows the formation of internal representations of the environment for navigation, and under visual guidance, surrounding space is generally accurately represented in relation to participants' action capabilities. However, internal spatial representations based on sound are likely coarser than for vision (Milne et al. 2014; Kolarik et al. 2016b). Studies have shown that blind individuals develop and primarily rely on egocentric spatial representations where the body is used as a means to center organization of the surrounding space (Corazzini et al. 2010; Iachini et al. 2014; Schinazi et al. 2016), and egocentric spatial representations could be used as a basis for navigation if the spatial location of a single sound source was used as a goal. However, a relatively coarse compressed auditory internal representation of distance could result in the perceived location of the goal being inaccurate or imprecise during initial path planning. This could affect locomotion by those with sight loss during wayfinding, leading to slower or less accurate movements, as the central nervous system (CNS) would have to compensate for the compression of perceived auditory space to reach the goal.

For farther sound sources, it is possible that the compression of perceived auditory distance associated with blindness provides an evolutionary advantage. Underestimation of the distance of farther sound sources, as occurs for sighted individuals, has been proposed to provide an additional "margin of safety" for navigating safely through the environment and avoiding obstacles using sound (Zahorik et al. 2005), similar to that resulting from the systematic underestimation of the time-to-arrival of approaching sound sources by sighted individuals (Ghazanfar et al. 2002). One possibility is that the greater-than-normal compression of perceived auditory distance for blind individuals reflects an adaptive bias, providing a selective advantage in preparing for contact with a farther sound source and in guiding locomotion. For example, perceiving a farther sound source to be closer than it actually is would promote an earlier response, which would be advantageous if the signal was threatening.

It is not currently known whether relative and absolute distance cues are processed by different neuronal mechanisms. Also, while many studies have investigated the cross-modal reorganization of visual brain regions that may underlie enhanced sound source localization by blind participants (reviewed by Collignon et al. 2009, and Voss et al. 2010), we are not aware of any studies that have investigated whether visual areas are recruited to process auditory distance following sight loss. Further work is needed to link the behavioral evidence for compressive distance judgments presented here and previously (Kolarik et al. 2013c) with neuronal mechanisms of auditory distance perception by blind participants.

The age of onset and duration of sight loss can affect auditory abilities (Voss et al. 2004; Wan et al. 2010) and can affect the extent of cross-modal recruitment in dorsal brain regions in response to auditory spatial information (Dormal et al. 2012). Age of onset of sight loss may also be a factor in the topographical representation of auditory space. For normally sighted listeners, it is possible that the representation of the auditory world is being constantly updated using calibration information from visual signals. When vision is lost, the representation of distance information based on hearing is not maintained and becomes more compressed. If this is so, then a compressed representation of auditory distance should be found even among late-onset blind listeners. Alternatively, the cross-modal calibration hypothesis (Gori et al. 2010) proposes that during development, information from a more accurate sense, such as vision, is used to calibrate another less accurate sense, such as hearing, and that once established the calibration does not need to be renewed or repeated. If this is so, the compression of auditory space should be present in early but not in late-blind listeners.

Previous work has shown that for relative distance tasks, blind listeners display better performance than sighted listeners (Ashmead et al. 1998; Voss et al. 2004), in both anechoic and reverberant virtual environments (Kolarik et al. 2013b), although this was not found by Cappagli et al. (2015). Such tasks do not require the participant to report where in space the sounds are perceived to be. Rather, the tasks depend on a comparison between the acoustic characteristics of the two stimuli, differing in physical characteristics such as level or $\mathrm{D} / \mathrm{R}$ values. Blind participants are better able to distinguish small differences in level or $\mathrm{D} / \mathrm{R}$ 
than sighted participants (Kolarik et al. 2013b), and this can explain their better performance in relative distance judgments. However, absolute judgments of distance depend on internal spatial representations of the external world without the benefit of any reference point or comparison stimuli. The results of the present study suggest that these representations are compressed among blind participants.

Taken together, the findings of the current study and those of Kolarik et al. (2013b) show that blind participants are better able than sighted participants to use small differences in acoustic cues to tell which of two sounds is closer, but are worse at reporting how far away those sound sources are. This parallels previous findings in the vertical dimension, showing that blind participants are often better than sighted participants in judging the relative positions of sound sources in elevation (Ashmead et al. 1998), but show deficits for absolute judgments of elevation (Lewald 2002b; Voss et al. 2015). The current results suggest that any auditory sensory enhancement that develops following severe visual loss does not also result in enhanced accuracy in judging the distance of sounds. Rather, blind people experience a compressed representation of auditory distance, at least when listening to a simulation of single sound sources in quiet anechoic or reverberant rooms. However, further work is needed to investigate the effect of visual loss on absolute distance perception in other situations that occur often in everyday life, such as when background noise and multiple sound sources are present. Although blind individuals are likely to constantly update their spatial representations via multiple sound sources when present, if this occurs within a representation of auditory distance that is compressed overall, accuracy may still be compromised compared to sighted listeners as the absolute distances of the reference sounds may be systematically underestimated in far space.

Acknowledgements We thank the editor Melvyn Goodale, and two anonymous reviewers for helpful comments that improved a previous version of the manuscript. This research was supported by MRC Grant G0701870 and the Vision and Eye Research Unit (VERU), Postgraduate Medical Institute at Anglia Ruskin University. We thank the participants, Camsight, The Norfolk \& Norwich Association for the Blind, Michael Stone for help and advice, and John Peters and George Oluwafemi for their assistance in data collection.

\section{Compliance with ethical standards}

Conflict of interest The authors declare that they have no conflict of interest.

Ethical approval All procedures performed in studies involving human participants were in accordance with the ethical standards of the institutional and/or national research committee and with the 1964 Helsinki Declaration and its later amendments or comparable ethical standards.
Open Access This article is distributed under the terms of the Creative Commons Attribution 4.0 International License (http://creativecommons.org/licenses/by/4.0/), which permits unrestricted use, distribution, and reproduction in any medium, provided you give appropriate credit to the original author(s) and the source, provide a link to the Creative Commons license, and indicate if changes were made.

\section{References}

Akeroyd MA, Gatehouse S, Blaschke J (2007) The detection of differences in the cues to distance by elderly hearing-impaired listeners. J Acoust Soc Am 121:1077-1089

Ashmead DH, Hill EW, Talor CR (1989) Obstacle perception by congenitally blind children. Atten Percept Psychophys 46:425-433

Ashmead DH, Wall RS, Ebinger KA, Eaton SB, Snook-Hill M, Yang X (1998) Spatial hearing in children with visual disabilities. Perception 27:105-122

Axelrod S (1959) Effects of early blindness: performance of blind and sighted children on tactile and auditory tasks. American Foundation for the Blind, New York

Bench J, Kowal A, Bamford J (1979) The BKB (Bamford-KowalBench) sentence lists for partially-hearing children. Br J Audiol 13:108-112

British Society of Audiology (2011) Pure-tone air-conduction and bone-conduction threshold audiometry with and without masking. British Society of Audiology, Reading

Brungart DS, Scott KR (2001) The effects of production and presentation level on the auditory distance perception of speech. J Acoust Soc Am 110:425-440

Calcagno ER, Abregú EL, Eguía MC, Vergara R (2012) The role of vision in auditory distance perception. Perception 41:175-192

Cappagli G, Cocchi E, Gori M (2015) Auditory and proprioceptive spatial impairments in blind children and adults. Dev Sci 18:1-12

Coleman PD (1963) An analysis of cues to auditory depth perception in free space. Psychol Bull 60:302-315

Collignon O, Voss P, Lassonde M, Lepore F (2009) Cross-modal plasticity for the spatial processing of sounds in visually deprived subjects. Exp Brain Res 192:343-358

Corazzini L, Tinti C, Schmidt S, Mirandola C, Cornoldi C (2010) Developing spatial knowledge in the absence of vision: allocentric and egocentric representations generated by blind people when supported by auditory cues. Psychol Belg 50:327-334

Da Silva JA (1985) Scales for perceived egocentric distance in a large open field: comparison of three psychophysical methods. Am J Psychol 98:119-144

Dormal G, Lepore F, Collignon O (2012) Plasticity of the dorsal "spatial" stream in visually deprived individuals. Neural Plast 2012:687659

Doucet ME, Guillemot JP, Lassonde M, Gagne JP, Leclerc C, Lepore F (2005) Blind subjects process auditory spectral cues more efficiently than sighted individuals. Exp Brain Res 160:194-202

Frenz H, Lappe M (2005) Absolute travel distance from optic flow. Vis Res 45:1679-1692

Gardner MB (1969) Distance estimation of $0^{\circ}$ or apparent $0^{\circ}$-oriented speech signals in anechoic space. J Acoust Soc Am 45:47-53

Gardner WG, Martin KD (1995) HRTF measurements of a KEMAR. J Acoust Soc Am 97:3907-3908

Ghazanfar AA, Neuhoff JG, Logothetis NK (2002) Auditory looming perception in rhesus monkeys. Proc Natl Acad Sci USA 99:15755-15757 
Gori M, Sandini G, Martinoli C, Burr D (2010) Poor haptic orientation discrimination in nonsighted children may reflect disruption of cross-sensory calibration. Curr Biol 20:223-225

Gori M, Sandini G, Martinoli C, Burr DC (2014) Impairment of auditory spatial localization in congenitally blind human subjects. Brain 137:288-293

Iachini T, Ruggiero G, Ruotolo F (2014) Does blindness affect egocentric and allocentric frames of reference in small and large scale spaces? Behav Brain Res 273:73-81

Jones B (1975) Spatial perception in the blind. Br J Psychol 66:461-472

Kolarik AJ, Cirstea S, Pardhan S (2013a) Discrimination of virtual auditory distance using level and direct-to-reverberant ratio cues. J Acoust Soc Am 134:3395-3398

Kolarik AJ, Cirstea S, Pardhan S (2013b) Evidence for enhanced discrimination of virtual auditory distance among blind listeners using level and direct-to-reverberant cues. Exp Brain Res 224:623-633

Kolarik AJ, Cirstea S, Pardhan S, Moore BCJ (2013c) An assessment of virtual auditory distance judgements among blind and sighted listeners. Proc Meet Acoust 19:050043

Kolarik AJ, Pardhan S, Cirstea S, Moore BCJ (2013d) Using acoustic information to perceive room size: effects of blindness, room reverberation time, and stimulus. Perception 42:985-990

Kolarik AJ, Moore BCJ, Zahorik P, Cirstea S, Pardhan S (2016a) Auditory distance perception in humans: a review of cues, development, neuronal bases and effects of sensory loss. Atten Percept Psychophys 78:373-395

Kolarik AJ, Scarfe AC, Moore BCJ, Pardhan S (2016b) An assessment of auditory-guided locomotion in an obstacle circumvention task. Exp Brain Res 234:1725-1735

Lehmann EA, Johansson AM (2008) Prediction of energy decay in room impulse responses simulated with an image-source model. J Acoust Soc Am 124:269-277

Lessard N, Pare M, Lepore F, Lassonde M (1998) Early-blind human subjects localize sound sources better than sighted subjects. Nature 395:278-280

Lewald J (2002a) Opposing effects of head position on sound localization in blind and sighted human subjects. Eur J Neurosci 15:1219-1224

Lewald J (2002b) Vertical sound localization in blind humans. Neuropsychologia 40:1868-1872

Lewald J (2013) Exceptional ability of blind humans to hear sound motion: implications for the emergence of auditory space. Neuropsychologia 51:181-186

Loomis JM, Klatzky RL, Philbeck JW, Golledge RG (1998) Assessing auditory distance perception using perceptually directed action. Atten Percept Psychophys 60:966-980

Macé MJM, Dramas F, Jouffrais C (2012) Reaching to sound accuracy in the peri-personal space of blind and sighted humans. In: Miesenberger K, Karshmer A, Penaz P, Zagler W (eds) Computers helping people with special needs: 13th international conference, ICCHP 2012. Springer, Linz, pp 636-643

Mershon DH, King LE (1975) Intensity and reverberation as factors in the auditory perception of egocentric distance. Atten Percept Psychophys 18:409-415

Milne JL, Goodale MA, Thaler L (2014) The role of head movements in the discrimination of 2-D shape by blind echolocation experts. Atten Percept Psychophys 76:1828-1837

Moore BCJ, Füllgrabe C, Stone MA (2011) Determination of preferred parameters for multichannel compression using individually fitted simulated hearing aids and paired comparisons. Ear Hear 32:556-568
O'Regan JK, Noë A (2001) A sensorimotor account of vision and visual consciousness. Behav Brain Sci 24:939-972

Otani M, Hirahara T, Ise S (2009) Numerical study on source-distance dependency of head-related transfer functions. J Acoust Soc Am 125:3253-3261

Philbeck JW, Loomis JM (1997) Comparison of two indicators of perceived egocentric distance under full-cue and reduced-cue conditions. J Exp Psychol Hum Percept Perform 23:72-85

Rice C (1970) Early blindness, early experience and perceptual enhancement. Res Bull Am Found Blind 22:1-22

Rowan D, Papadopoulos T, Edwards D, Holmes H, Hollingdale A, Evans L, Allen R (2013) Identification of the lateral position of a virtual object based on echoes by humans. Hear Res 300:56-65

Schinazi VR, Thrash T, Chebat DR (2016) Spatial navigation by congenitally blind individuals. Wiley Interdiscip Rev Cogn Sci 7:37-58

Teng S, Puri A, Whitney D (2012) Ultrafine spatial acuity of blind expert human echolocators. Exp Brain Res 216:483-488

Turano KA, Yu D, Hao L, Hicks JC (2005) Optic-flow and egocentricdirection strategies in walking: central vs peripheral visual field. Vis Res 45:3117-3132

Vercillo T, Milne JL, Gori M, Goodale MA (2015) Enhanced auditory spatial localization in blind echolocators. Neuropsychologia 67:35-40

Vercillo T, Burr D, Gori M (2016) Early visual deprivation severely compromises the auditory sense of space in congenitally blind children. Dev Psychol 52:847

Voss P, Zatorre RJ (2012) Organization and reorganization of sensorydeprived cortex. Curr Biol 22:168-173

Voss P, Lassonde M, Gougoux F, Fortin M, Guillemot JP, Lepore F (2004) Early- and late-onset blind individuals show supra-normal auditory abilities in far-space. Curr Biol 14:1734-1738

Voss P, Collignon O, Lassonde M, Lepore F (2010) Adaptation to sensory loss. Wiley Interdiscip Rev Cogn Sci 1:308-328

Voss P, Lepore F, Gougoux F, Zatorre RJ (2011) Relevance of spectral cues for auditory spatial processing in the occipital cortex of the blind. Front Psychol 2:48

Voss P, Tabry V, Zatorre RJ (2015) Trade-off in the sound localization abilities of early blind individuals between the horizontal and vertical planes. J Neurosci 35:6051-6056

Wan CY, Wood AG, Reutens DC, Wilson SJ (2010) Early but not lateblindness leads to enhanced auditory perception. Neuropsychologia 48:344-348

Wanet M, Veraart C (1985) Processing of auditory information by the blind in spatial localization tasks. Atten Percept Psychophys 38:91-96

World Health Organization (1989) Tenth Revision of the International Classification of Disease. World Health Organization, Geneva

Zahorik P (2001) Estimating sound source distance with and without vision. Optom Vis Sci 78:270-275

Zahorik P (2002a) Assessing auditory distance perception using virtual acoustics. J Acoust Soc Am 111:1832-1846

Zahorik P (2002b) Direct-to-reverberant energy ratio sensitivity. J Acoust Soc Am 112:2110-2117

Zahorik P, Brungart DS, Bronkhorst AW (2005) Auditory distance perception in humans: a summary of past and present research. Acta Acust United Acust 91:409-420

Zwiers M, Van Opstal A, Cruysberg J (2001) A spatial hearing deficit in early-blind humans. J Neurosci 21:141-145 